\title{
A unidade retórica de Metodologia em artigos empíricos da cultura disciplinar da área de Psicologia: uma investigação sociorretórica
}

\section{The Rhetorical Unit of Methods in Empirical Academic Articles of Psychology Area: A Socio and Rhetorical Investigation}

Cibele Gadelha Bernardino*

*Universidade Estadual do Ceará (UECE), Fortaleza, Ceará / Brasil cibele.gadelha@uece.com

Nícollas Oliveira Abreu**

**Universidade Estadual do Ceará (UECE), Fortaleza, Ceará / Brasil nicollas.abreu@aluno.uece.br

RESUMO: Este artigo tem como objetivo descrever como a área de Psicologia constrói e entende a unidade de Metodologia em artigos acadêmicos empíricos. Como aporte teórico, nos embasamos em Swales (1990) quanto às concepções que envolvem os gêneros acadêmicos e também em relação à sua metodologia CARS. No que concerne ao estudo das culturas disciplinares, fundamentamonos na pesquisa de Hyland (2000). Desse modo, este estudo, que é compreendido como uma pesquisa de natureza exploratório-descritiva, apresenta um corpus de trinta exemplares de artigos acadêmicos, que são originados de dez periódicos da área de Psicologia, indexados no banco de dados WEBQUALIS da Capes. De acordo com esta pesquisa, verificamos que a seção de Metodologia é percebida como uma unidade bem detalhada, apresentando informações relacionadas ao tamanho e ao perfil da amostra, descrições dos materiais ou instrumentos utilizados, procedimentos de pesquisa, dados relacionados à aprovação das pesquisas por comitês de ética e descrição de análises dos dados.

PALAVRAS-CHAVE: descrição sociorretórica; cultura disciplinar; área de psicologia; artigo empírico; seção de metodologia.

ABSTRACT: This research aims at describing how the Psychology area produces and understands the Methods unit in empirical academic articles. As a theoretical basis, we relied on Swales (1990) for the concepts involving academic genres and its Create a Research Space methodology. Hyland's (2000) research was used as basis for the study of disciplinary cultures. Thus, our study, 
classified as an exploratory and descriptive research, has a corpus consisting of 30 copies of academic articles from 10 journals in the Psychology area, indexed in the WEBQUALIS platform from the Capes database. In our study we verified that the Methods sections had a very detailed section, providing information related to the sample size and profile, descriptions of materials or instruments used, research procedures, data related to the approval by research ethics committees and description of data analysis.

KEYWORDS: Social and rhetorical description; disciplinary culture; Psychology area; empirical article; methods section.

\section{Introdução}

Os estudos sobre análise de gêneros, conforme constata Bhatia (2009), têm se tornado cada vez mais populares nos últimos anos. Na área de Linguística Aplicada, pesquisas que investigam os gêneros acadêmicos vêm ganhando ainda maior força, com destaque para o artigo acadêmico que, conforme Swales (1990), Hyland (2000) e Motta-Roth e Hendges (2010), constitui-se como o gênero de maior relevância para a produção, divulgação e refutação de pesquisas.

Apesar da reconhecida importância do artigo entre os gêneros acadêmicos, temos percebido que, muitas vezes, os alunos de graduação e pós-graduação recebem recomendações generalizadas para a produção desse gênero, seja na forma de orientações dadas pelos docentes, seja por meio de materiais didáticos que versam sobre essa temática. Pinheiro (2016) e Lima e Abreu (2017) constataram que alguns manuais voltados para o ensino de Metodologia Científica e aqueles de orientação da escrita científica têm desconsiderado aspectos intrínsecos aos distintos campos do conhecimento na produção do gênero artigo acadêmico, propondo, assim, uma forma homogênea para a produção escrita de exemplares de artigos para diferentes áreas.

Quando discutimos sobre a investigação de gêneros acadêmicos, torna-se imprescindível refletir que, para compreender como ocorrem sua produção, circulação e seu consumo, é preciso conhecer aspectos culturais que lhes são próprios. Seguindo essa lógica, encontramos em Hyland (2000) argumentos que justificam a necessidade de exploração dos contextos culturais aos quais os gêneros acadêmicos estão vinculados para que assim seja possível entender como esses gêneros são construídos e compreendidos nas mais variadas culturas disciplinares. 
Ao partirmos dessa premissa teórica, acreditamos que o diálogo entre a perspectiva sociorretórica de Swales (1990) e os estudos de Hyland (2000) sobre a escrita acadêmica em diferentes culturas disciplinares é um terreno fértil para as atuais pesquisas preocupadas com o letramento acadêmico. Em relação ao gênero artigo acadêmico, apontamos algumas pesquisas que convergem para essa articulação teórica, como: a investigação de Costa (2015), que comparou a organização sociorretórica de artigos completos das áreas de Linguística e Medicina; o estudo de Pacheco (2016), que se debruçou sobre a configuração sociorretórica de artigos originais completos da área de Nutrição; e a pesquisa de Abreu (2016), que investigou a descrição sociorretórica em artigos empíricos completos da área de Psicologia.

Com o propósito de descrever o gênero artigo acadêmico a partir da cultura disciplinar da área de Psicologia, concatenando as concepções de Swales (1990) e Hyland (2000) no que concerne ao referido gênero, este artigo, vinculado ao grupo de pesquisa Discurso, Identidade e Letramento Acadêmicos (Dileta), ${ }^{1}$ objetiva investigar e descrever a unidade de Metodologia ${ }^{2}$ em artigos empíricos com base nas características da cultura disciplinar dessa área, procurando compreender como as crenças e os valores da referida cultura influenciam a produção e compreensão do gênero em questão.

\section{Fundamentação teórica}

\subsection{Definindo o gênero artigo acadêmico}

Primeiramente, vale ressaltar que, neste trabalho, empreendemos uma análise de gênero, a partir da qual, de acordo com Bhatia (2009), é possível estudar o comportamento linguístico/discursivo tanto em âmbitos acadêmicos como em profissionais. Em relação à concepção do termo

\footnotetext{
${ }^{1}$ Para mais informações, acesse < https://bit.ly/2IOQ0Ec $>$.

${ }^{2}$ Este artigo apresenta parte dos resultados de uma pesquisa maior vinculada ao projeto de pesquisa Práticas discursivas em comunidades disciplinares acadêmicas, que investiga as variações nas configurações sociorretóricas do gênero artigo acadêmico em diferentes áreas disciplinares, entre elas a de Psicologia. A pesquisa em questão (Abreu, 2016) já descreveu todas as seções retóricas de exemplares do gênero artigo acadêmico do campo da Psicologia; entretanto, por conta da necessidade de maior espaço para a discussão desses dados, apenas descrevemos aqui a unidade de Metodologia.
} 
gênero, nos embasamos teoricamente em Swales (1990, p. 58). Consoante o autor,

um gênero compreende uma classe de eventos comunicativos, cujos membros compartilham um conjunto de propósitos comunicativos. Estes propósitos são reconhecidos pelos membros especialistas da comunidade discursiva e assim constituem a razão do gênero. Esse raciocínio molda a estrutura esquemática do discurso, influencia e restringe a escolha de conteúdo e estilo. O propósito comunicativo é um critério privilegiado e opera para manter o escopo de um gênero aqui concebido como uma ação retórica comparável. Em adição ao propósito, exemplares de um gênero exibem vários padrões de similaridade em termos de estrutura, estilo, conteúdo e público-alvo. Se todas as expectativas forem realizadas, o exemplar será reconhecido como prototípico pelos membros experientes da comunidade discursiva (tradução nossa). ${ }^{3}$

Os estudos de Swales (1990; 2004), de maneira geral, tratam o artigo acadêmico como o gênero mais utilizado na academia, nos levando a crer na relevância desse gênero para a educação superior. Motta-Roth e Hendges (2010) corroboram o raciocínio do autor, reconhecendo o artigo como meio de produção e divulgação de conhecimentos oriundos de pesquisas. Ainda sobre o gênero artigo acadêmico, as autoras afirmam haver diferentes tipos, enfatizando os teóricos, os de revisão de literatura e os experimentais.

Para Swales (2004), o artigo de revisão discute a literatura existente, que é, muitas vezes, finalizada com uma avaliação global. Com base em Bernardino (2006), entendemos o artigo teórico como aquele que elabora uma discussão teórica, não recorrendo necessariamente à investigação de

\footnotetext{
${ }^{3}$ No original: "A genre comprises a class of communicative events, the members of which share some set of communicative purposes. These purposes are recognized by the expert members of the parent discourse community, and thereby constitute the rationale for the genre. This rationale shapes the schematic structure of the discourse and influence and constrains choice of content and style. Communicative purpose is both a privileged criterion and one that operates to keep the scope of a genre as here conceived narrowly focused on comparable rhetorical action. In addition to purpose, exemplars of a genre exhibit various patterns of similarity in terms of structure, style, content and intended audience. If all high probability expectations are realized, the exemplar will be viewed as prototypical by the parent discourse community".
} 
um corpus com o objetivo de analisar dados. $\mathrm{O}$ artigo do tipo experimental ${ }^{4}$ - foco da análise desta pesquisa - conforme Bernardino (2006), apresenta como propósito analisar e discutir dados originados de qualquer natureza, estando presentes em sua configuração retórica as unidades de Resultados e Discussão, que podem ser construídas de maneira conjunta ou aparecer como unidades avulsas. A autora afirma que informações metodológicas também são apresentadas nesse tipo de artigo e podem vir ou não em uma unidade retórica destacada.

Depois de conceituarmos as diferentes variações de artigos acadêmicos, voltemo-nos para a análise desse objeto de pesquisa. Para a investigação do gênero artigo acadêmico, concordamos com o que argumenta Hyland (2000) no que diz respeito à necessidade de estudar o gênero associado ao estudo da cultura disciplinar, posto que há, entre as diferentes culturas, variações disciplinares. Nessa perspectiva, apresentamos no tópico seguinte o conceito de cultura disciplinar.

\subsection{Entendendo a ideia de cultura disciplinar}

De acordo com Bhatia (2009, p. 179), os gêneros são construídos e mantidos por práticas sociais, e é a partir desses gêneros que os membros de comunidades acadêmicas interagem entre si. Hyland (2000) argumenta que o discurso disciplinar é um amplo recurso de informação quanto às práticas sociais existentes na academia. Essas práticas sociais, por sua vez, refletem as características das distintas culturas disciplinares. Podemos dizer, assim, que existe uma relação de mão dupla entre crenças, valores e epistemologias das áreas disciplinares e sua forma de construir os gêneros acadêmicos.

Caracterizando as culturas disciplinares, Hyland (2000) conceitua as disciplinas como sistemas em que as crenças e práticas dialogam com metodologias, convenções, normas e terminologias. Vale ressaltar, portanto, que as culturas disciplinares podem se diferenciar quantos aos objetivos, questões cognitivas e formas de comportamento. Essas características podem ser observadas nos gêneros, já que, de acordo com o autor, eles são sensíveis a variações disciplinares.

Para Hyland (2000, p. 1-2, tradução nossa),

\footnotetext{
${ }^{4} \mathrm{O}$ artigo experimental, na área de Psicologia, é denominado artigo empírico, nomenclatura que adotaremos a partir deste momento para designar o nosso objeto de estudo.
} 
estudar as interações sociais presentes na escrita acadêmica não é somente ver como escritores em diferentes disciplinas produzem conhecimento, é também revelar algo sobre os comportamentos sociais aprovados, crenças epistêmicas e estruturas institucionais de comunidades acadêmicas. ${ }^{5}$

O autor defende que as culturas disciplinares são formadas por pluralidades de crenças e práticas, que podem implicar diferentes maneiras de produzir um gênero acadêmico, como também o modo de entendê-lo e utilizá-lo.

Desse modo, acreditamos que os estudos de Hyland (2000) contribuem para a percepção de que os gêneros acadêmicos devem ser investigados a partir das culturas disciplinares, possibilitando a compreensão de como as crenças e valores sociais influenciam a construção desses gêneros e de como os gêneros alimentam tais características da cultura. Acreditamos que essa visão de Hyland dialoga de forma produtiva com a proposta contextual de análise de gêneros estabelecida por Askehave e Swales (2009), uma vez que permite que a descrição da configuração composicional, assim como das características linguísticas dos gêneros possam ser caracterizadas e analisadas à luz do filtro da cultura, do grupo social que usa os gêneros.

De uma forma geral, a proposta de análise de Swales (1990), da qual é oriunda a metodologia CARS, é reconhecida como um modelo de descrição de introduções de artigos acadêmicos, porém entendemos esta proposta como mais do que uma descrição da configuração composicional de introduções. Em nossa compreensão, essa proposta é uma metodologia de análise de gêneros de variados domínios discursivos, que toma como ponto de partida o critério da prototipicidade das unidades retóricas recorrentes que desenham a configuração composicional dos exemplares prototípicos dos gêneros. Cabe ressaltar que essa configuração retórica está diretamente relacionada aos propósitos comunicativos que regulam o funcionamento dos gêneros, correspondendo ao que Swales (1990) chama de "razão subjacente ao gênero". Em defesa dessa concepção, lembramos que há diversas pesquisas que se apropriaram da Metodologia CARS para a análise

\footnotetext{
${ }^{5}$ No original: "study the social interactions expressed through academic writing is not only to see how writers in different disciplines go about producing knowledge, it is also to reveal something of the sanctioned social behaviors, epistemic beliefs, and institutional structures of academic communities".
} 
de gêneros variados. Apresentaremos, a seguir, as propostas ${ }^{6}$ de Nwogu (1997), Oliveira (2003) e Costa (2015) para a descrição da configuração composicional do gênero artigo acadêmico com base na Metodologia CARS de John Swales.

\subsection{Apresentando as propostas retóricas da seção de Metodologia}

Neste estudo, analisamos e descrevemos como é organizada a configuração sociorretórica na seção de Metodologia a partir de trinta exemplares de artigos empíricos de dez periódicos nacionais da área de Psicologia, com estratos de B2 a A1. Vale ressaltar que essa unidade retórica não foi descrita por Swales (1990) na Metodologia CARS. Conforme discutimos, a proposta do autor - voltada para a seção de Introdução - inspirou muitas outras pesquisas, que desenvolveram diferentes configurações retóricas para distintas seções retóricas.

Neste estudo, utilizamos principalmente a proposta retórica de Oliveira (2003), que se aproximou dos dados obtidos a partir da investigação do nosso corpus. Em sua pesquisa, formulou a descrição da seção de Metodologia em artigos acadêmicos da área de Linguística Aplicada. Observemos, a seguir, como é organizada a proposta da autora por meio de seus movimentos ${ }^{7} \mathrm{e}$ passos:

\footnotetext{
${ }^{6}$ As propostas retóricas de Nwogu (1997) e Costa (2015), também contempladas neste estudo, por uma questão de limite de espaço, serão discutidas apenas na seção de Resultados e Discussão.

${ }^{7}$ Para Swales (2004), os movimentos (moves) são unidades retóricas que realizam uma função comunicativa no discurso, seja falado ou escrito. Quanto aos passos (steps), podemos caracterizá-los como unidades menores que compõem os movimentos e que possuem funções comunicativas específicas associadas ao objetivo dos movimentos.
} 
QUADRO 1 - Descrição retórica da unidade de Metodologia em artigos acadêmicos da área de Linguística Aplicada ${ }^{8}$

\begin{abstract}
MOVIMENTO 1-Descrição do corpus ou participantes da pesquisa
PASSO 1: Especificação do tamanho da amostra (tamanho do corpus ou número de participantes)
\end{abstract}

PASSO 2: Especificação do perfil dos participantes

PASSO 2A: Especificação do sexo e idade

PASSO 2B: Especificação do nível de escolaridade (estudantes, professores, etc.)

PASSO 2C: Especificação da subárea a que os participantes pertencem

PASSO 2D: Especificação do nível de conhecimento dos participantes na língua ou tópico que está sendo investigado pela pesquisa

$\mathrm{OU}$

PASSO 3: Especificação do corpus selecionado

MOVIMENTO 2 - Descrição dos materiais ou instrumentos utilizados na coleta dos dados

MOVIMENTO 3-Descrição dos procedimentos

MOVIMENTO 4 - Descrição da análise dos dados

Fonte: Oliveira (2003, p. 153).

De acordo com Oliveira (2003), o movimento 1 - Descrição do corpus ou participantes da pesquisa - caracteriza, em geral, a informação que primeiramente aparece na seção de Metodologia. Para a autora, o autor do artigo costuma apresentar o tamanho do corpus analisado ou o número de pessoas que participaram da pesquisa.

$\mathrm{Na}$ sequência, o autor da pesquisa apresenta o perfil dos participantes, se detendo a descrever informações como sexo, idade, nível de escolaridade e até a subárea ou nível a que pertencem. Ademais, podem aparecer no artigo dados acerca do nível de conhecimento do participante, que pode estar relacionado ao tópico investigado na pesquisa ou ao conhecimento da língua-alvo.

\footnotetext{
${ }^{8}$ Estão destacados em negrito, na descrição retórica, apenas os movimentos e passos que alcançaram a recorrência no corpus desta pesquisa. Consideramos recorrência uma frequência igual ou superior a $50 \%$.
} 
O movimento 2 - Descrição dos materiais ou instrumentos utilizados na coleta de dados - discorre sobre diferentes instrumentos ou materiais empregados no estudo, como questionários, entrevistas, programas de computador, entre outros. Vale salientar que esses materiais ou instrumentos podem ser citados tanto em pesquisas qualitativas como quantitativas.

No movimento 3 - Descrição dos procedimentos -, conforme Oliveira (2003), os autores podem escrever como as informações foram coletadas, mencionando os instrumentos ou materiais utilizados. Por fim, no movimento 4 - Descrição da análise de dados - há a descrição de como serão feitas a análise e a interpretação dos dados.

Após evidenciarmos a principal proposta teórico-metodológica que norteia este estudo, vejamos, a seguir, os procedimentos metodológicos desta pesquisa.

\section{Percurso metodológico}

Neste estudo, desenvolvemos uma pesquisa exploratório-descritiva que empreende uma análise de gênero. Para a compreensão do gênero artigo acadêmico empírico da cultura disciplinar da área de Psicologia, procuramos entender as especificidades dessa área no que concerne à maneira de produção e compreensão do gênero em questão. Por meio da investigação da relação entre a cultura disciplinar e a recorrência dos movimentos e passos retóricos do gênero investigado, descrevemos como a esfera da Psicologia produz o artigo acadêmico empírico. ${ }^{9}$

Quanto ao corpus desta pesquisa, dispomos de trinta exemplares do gênero artigo acadêmico empírico da cultura disciplinar do campo da Psicologia, ${ }^{10}$ publicados entre os anos de 2012 e 2015, em dez periódicos da área, a saber: Estudos e Pesquisas em Psicologia; Psicologia e Sociedade; Saúde e Sociedade; Saúde em Debate; Psicologia: Teoria e Pesquisa; Fractal: Revista de Psicologia; Temas em Psicologia; Psicologia em Pesquisa; Estudos de Psicologia; e Psico-USF. No que diz respeito à estratificação dos periódicos, o corpus varia do Qualis B2

\footnotetext{
${ }^{9}$ Este estudo é amparado pelo Comitê de Ética em Pesquisa (CEP) da Universidade Estadual do Ceará (Uece), conforme o processo nº 0671978/2014.

${ }^{10}$ Os exemplares de artigos do corpus foram etiquetados em Artigo Acadêmico Empírico de Psicologia (AAEP), que foram numerados de 1 a 30.
} 
ao A1, de acordo com Qualis Periódicos da Capes (2014). ${ }^{11}$ Os periódicos foram selecionados de acordo com os critérios de recorte temporal e do indicador Qualis da Capes supramencionado.

Para a análise do corpus, utilizamos como fundamentação as descrições da seção de Metodologia propostas por Nwogu (1997), Oliveira (2003) e Costa (2015). Contamos também com as orientações para a produção da seção de Metodologia descritas pelo manual da American Psychological Association - APA (2010), um guia que apresenta estilo de escrita para escritores, editores, estudantes, professores e pesquisadores de áreas de ciências sociais e comportamentais, como a Psicologia. Esse manual orienta quanto aos aspectos do processo de escrita, como estilo e escolhas lexicais, trazendo, ainda, recomendações sobre como cada seção do artigo deve ser produzida. Depois de caracterizarmos o corpus e o processo de análise, evidenciamos que este estudo parte da conjectura defendida por Hyland (2000), ${ }^{12}$ de que as diferentes culturas disciplinares constituem de maneiras distintas os gêneros acadêmicos.

Com o propósito de descrevermos a cultura disciplinar da área de Psicologia, investigamos, neste trabalho, artigos, livros e sites que retratam a origem desse campo no Brasil, a formação do profissional e informações voltadas à pós-graduação da área de Psicologia no país. Também consultamos sites dos conselhos regionais e Federal de Psicologia para entendermos a maneira de organização dos profissionais. Em outro momento, averiguamos os relatórios da Capes, que discutem sobre regulamentos e avaliações dessa esfera no Brasil. Na sequência, analisamos as orientações dos periódicos do corpus para a produção do gênero artigo acadêmico. Por fim, na última etapa

\footnotetext{
${ }^{11}$ É fundamental ressaltar que, nesta pesquisa, discutiram-se os dados do Qualis 2014, visto que era o mais recente no momento em que os dados foram analisados. No entanto, destacamos que já está em vigor o Qualis 2016.

${ }^{12} \mathrm{O}$ autor em questão, apesar de apresentar um rico aporte teórico acerca do conceito de cultura disciplinar, não fornece um percurso metodológico que possibilite a investigação do conjunto de crenças, valores e propósitos pertencentes a determinada área. Por meio dessa constatação, este artigo e os demais estudos vinculados ao grupo de pesquisa em Discurso, Identidade e Letramento Acadêmicos (Dileta/Uece) e ao projeto Práticas Discursivas em Comunidades Disciplinares Acadêmicas visam ao desenvolvimento de um percurso metodológico que permita a descrição de culturas disciplinares.
} 
da investigação, buscamos compreender o olhar dos membros experientes ${ }^{13}$ da área quanto à produção e compreensão do referido gênero. Para isso, contamos com a colaboração de doze membros experientes em dois programas de pós-graduação na área de Psicologia situados na cidade de Fortaleza (CE). Dentre os doze professores/pesquisadores consultados, oito responderam a uma entrevista realizada pessoalmente, e os outros quatro responderam questionários via e-mail. As entrevistas e os questionários foram conduzidos a partir da elaboração de um roteiro direcionado a investigar a produção acadêmica de artigos na área analisada. Ressaltamos que a descrição do gênero artigo, com base nas orientações dos periódicos do corpus e da compreensão dos membros experientes, nos possibilitou analisar a configuração sociorretórica da unidade de Metodologia dos artigos acadêmicos da cultura disciplinar da área de Psicologia.

Evidenciamos que a análise dos dados tem base qualitativa e quantitativa. Qualitativa por considerar inicialmente os dados referentes às crenças e valores da cultura disciplinar; quantitativa no que diz respeito ao fato de identificarmos as unidades informacionais presentes nos artigos com o propósito de apurarmos quais são as mais recorrentes (igual ou acima de $50 \%$ de frequência no corpus) e, por consequência, as mais prototípicas para a caracterização da configuração retórica da unidade. É importante sublinhar que esse momento do levantamento quantitativo parte dos dados qualitativos oriundos da descrição da cultura disciplinar.

Desse modo, articulamos os achados da análise da cultura disciplinar com a descrição proposta por Oliveira (2003) para analisar as unidades retóricas, investigando sobre como são elaborados os movimentos e passos do artigo empírico na área de Psicologia. Vejamos, a seguir, a descrição da cultura disciplinar dessa esfera e sua articulação com a descrição sociorretórica do artigo acadêmico empírico em Psicologia.

\footnotetext{
${ }^{13}$ Professores/pesquisadores que integram o corpo docente de um programa de pósgraduação na área de Psicologia, visto que são os membros que regularmente realizam a produção de artigos e apresentam fluxo contínuo de publicações em periódicos relevantes para o campo de atuação, fato que nos permite compreender, a partir das suas experiências, como ocorre a produção de pesquisa no âmbito acadêmico.
} 


\section{Resultados e Discussões}

\subsection{Caracterizando a cultura disciplinar da área de Psicologia no Brasil}

De acordo com o Conselho Regional de Psicologia da $6^{a}$ região CRPSP (2011), a Psicologia é reconhecida como uma ciência capacitada a elaborar práticas, técnicas e teorias que orientem e integrem o processo de desenvolvimento humano projetado pela nova ordem político-social. Como subdivisões da área, são estabelecidos distintos campos de atuação, entre eles: clínico, educacional e social.

Para o Conselho Federal de Psicologia (CFP), ${ }^{14}$ o psicólogo é concebido como o profissional que utiliza métodos e técnicas psicológicas com o propósito tanto de identificar como de orientar fatores determinantes das ações e dos sujeitos, investigando processos intrapessoais e relações interpessoais para compreender o comportamento humano, de maneira individual ou grupal, em âmbitos de diversas naturezas em que ocorram essas relações. Segundo o Conselho Regional de Psicologia da $6^{a}$ região CRPSP (2011), a década de 1950 foi considerada relevante para a área no Brasil, posto que muitos acontecimentos viabilizaram o desenvolvimento da Psicologia no país, como: o primeiro Congresso Brasileiro de Psicologia; o primeiro anteprojeto acerca da formação e regulamentação da profissão; a fundação de cursos de Psicologia na Faculdade de Filosofia, Ciências e Letras da Universidade de São Paulo (USP) e na Pontifícia Universidade Católica de São Paulo (PUC-SP), Minais Gerais (PUC-Minas) e Rio de Janeiro (PUCRio). Em 1958, é apresentado o Projeto de Lei no 3.825, que evidencia a regulamentação da profissão de psicólogo no Brasil. Quatro anos depois, em 1962, é decretada a Lei no 4.119, que apresenta o primeiro diploma legal sobre cursos de formação para psicólogos e, ainda, estabelece um currículo mínimo para a formação desse profissional (SOARES, 2010).

Em relação ao primeiro curso de pós-graduação em Psicologia no Brasil, consoante Féres-Carneiro (2007), em março de 1966, no Rio de Janeiro, surge o primeiro curso de mestrado do país na área, ofertado pelo Departamento de Psicologia da PUC-Rio. Em 1971, é fundado o Conselho Federal de Psicologia - sancionado pela Lei no 5.766, que também permitiu criar os conselhos regionais de psicologia - órgão responsável por significativas mudanças organizacionais e conquistas nesse campo.

${ }^{14}$ Disponível em: <https://bit.ly/2lPpph6>. Acesso em: 23 mar. 2016. 
Com o intuito de apreciarmos informações pertinentes no que concerne à pós-graduação da área de Psicologia no país, discutimos os dados do documento de área da Capes (2013a), de acordo com o qual a área de Psicologia é composta por 73 programas de pós-graduação, que, por sua vez, são compostos por 47 cursos de doutorado, 71 de mestrado acadêmico e 2 de mestrado profissional (aprovados em 2012), totalizando 120 cursos de pósgraduação. A metade dos programas de pós-graduação está situada na região Sudeste do Brasil - quase um terço no estado de São Paulo -, em segundo e terceiro lugar, respectivamente, vêm as regiões Nordeste e Sul. Em dados percentuais, enquanto a região Sudeste concentra 50\% dos programas de pós-graduação em Psicologia, a região norte possui apenas 5\%.

A partir de 1975, a pós-graduação no Brasil tem sido orientada pelos Planos Nacionais de Pós-Graduação (PNPG). Na sua sexta edição, o PNPG projeta ações para uma década (2011-2020). Os planos são voltados para a elaboração de estratégias na pós-graduação para que metas importantes para o desenvolvimento econômico e social do país sejam atingidas. Uma das metas desse plano é a redução das assimetrias regionais. Para que isso ocorra, deve ser fomentado o crescimento desses cursos nas regiões Norte e Centro-Oeste, que apresentam menor quantidade de programas, sendo necessárias ações da área para fortalecer os cursos de mestrado para que, consequentemente, surjam novos cursos de doutorado. Destarte, com a maior distribuição de programas e aumento da formação de mestres e doutores, esses acontecimentos poderão contribuir para o crescimento da área de Psicologia no país.

Dando continuidade à descrição da cultura disciplinar da área de Psicologia, analisamos as orientações dos periódicos do corpus desta pesquisa no que tange à elaboração dos artigos acadêmicos. Neste trabalho, foram investigados dez periódicos de circulação nacional. Dentre estes, quatro seguem integralmente as orientações do manual da American Psychological Association - APA (2010), a conhecer: Estudos e Pesquisas em Psicologia (2016), Psicologia: Teoria e Pesquisa (2016), Temas em Psicologia (2016) e Psico-USF (2016). Duas revistas utilizam o manual da APA (2010) com adaptações dos próprios periódicos: Estudos de Psicologia (2016) e Psicologia e Sociedade (2016). As revistas Fractal, Psicologia em Pesquisa, Saúde em Debate e Saúde e Sociedade apresentam raras ou nenhuma orientação para a elaboração das unidades retóricas dos artigos. 
Conforme orienta o manual da APA (2010), na produção da seção de Metodologia devem ser descritos detalhes de como o estudo foi realizado, já que é a partir dessa unidade que o leitor pode avaliar a adequação dos métodos empregados. É preciso apresentar também a identificação de subseções, os procedimentos para a coleta da amostra, as características dos participantes e os instrumentos e materiais utilizados na pesquisa. Em relação às subseções, defende-se que é conveniente dividir a seção de Metodologia em subseções, posto que cada divisão aponta uma característica específica, como a descrição da amostra, instrumentos/materiais utilizados ou procedimentos da pesquisa.

A APA (2010) aponta a importância de apresentar como e quantos participantes foram selecionados e fazem parte da amostra e os locais em que os dados foram coletados. Ainda em relação à amostra do estudo, o manual afirma que identificá-los é essencial, trazendo informações relacionadas à idade e ao sexo dos participantes. Dados sobre os materiais e instrumentos aplicados na pesquisa também são essenciais na composição dessa unidade retórica. São fundamentais informações acerca dos métodos empregados para a coleta de dados, como entrevistas, observações e questionários.

No que diz respeito às orientações do periódico para a elaboração dessa seção, o periódico Psicologia: Teoria e Pesquisa (2016) orienta que é imprescindível apresentar as subseções de descrição da amostra, instrumentos, materiais, equipamentos e procedimentos empregados para o andamento da pesquisa. O periódico Temas em Psicologia (2016), por sua vez, indica que nessa unidade devem ser contemplados os seguintes subtópicos: participantes, instrumentos, procedimentos de coleta de dados, procedimentos de análise de dados e procedimentos éticos.

A revista Psico-USF (2016) é breve ao afirmar que a Metodologia precisa apontar os participantes envolvidos na pesquisa, os instrumentos utilizados e os procedimentos do estudo. Já a Estudos de Psicologia (2016), que é mais detalhista no que tange à descrição de como essa unidade deve ser elaborada, indica que a seção de Metodologia deve incluir informações consistentes sobre a amostra, instrumentos e procedimentos utilizados. Além disso, o periódico ainda informa que, ao final dessa unidade, deve ser apresentada uma informação clara quanto ao atendimento sobre os procedimentos éticos adotados no estudo e número do processo aprovado por comitê de ética. 
Após descrevermos as orientações da APA (2010) e dos periódicos investigados no corpus desta pesquisa, nos debruçamos sobre as informações obtidas a partir das entrevistas e questionários com os membros experientes da cultura disciplinar da área de Psicologia para entendermos como eles produzem e compreendem a seção de Metodologia em artigos acadêmicos empíricos. Para o colaborador $2,{ }^{15}$ a seção de Metodologia não deve ser repetitiva, devendo ser direta a forma de apresentação dos métodos utilizados na pesquisa. Isso nos leva a crer que, nessa unidade, valoriza-se a concisão. O colaborador 3 justifica que essa unidade deve caracterizar a amostra e o referencial da pesquisa.

Segundo o colaborador 8, essa seção é indispensável para a composição do artigo acadêmico, pois caracteriza "como o trabalho foi feito". O pesquisador, mais criterioso, argumenta que, nessa seção, devem ser esclarecidos todos os passos do estudo, procedimentos, critérios de inclusão, instrumentos utilizados e quem os elaborou, como a coleta foi realizada, como os dados foram analisados e quais as características da amostra.

Para o colaborador 12, é relevante evidenciar o método de geração de análise de dados, aspectos éticos, descrição das características dos participantes envolvidos, instrumentos e procedimentos. O colaborador 10 enfatiza que é imprescindível que a Metodologia esteja adequada ao objeto de estudo. O pesquisador também sugere que deve haver subsídios para uma boa compreensão e interpretação da pesquisa.

Dessa maneira, por meio das informações originadas a partir da investigação da cultura disciplinar da área de Psicologia, pode-se entender como essa área produz e compreende a seção de Metodologia no artigo acadêmico empírico. A descrição da configuração sociorretórica da unidade de Metodologia dos artigos acadêmicos empíricos será realizada com base nesses dados. Sigamos, agora, para análise da unidade retórica de Metodologia de artigos acadêmicos empíricos da área de Psicologia.

\footnotetext{
${ }^{15}$ Refere-se aos colaboradores entrevistados nesta pesquisa, que são enumerados com uma contagem que vai de 1 a 12 . Os colaboradores de 1 a 8 responderam as entrevistas, enquanto os de 9 a 12 contribuíram por meio de questionários.
} 


\subsection{Descrevendo a seção de Metodologia ${ }^{16}$ em artigos empíricos da área de Psicologia}

A unidade de Metodologia, nos artigos empíricos da área de Psicologia, apresentou recorrência de 100\%, ou seja, em todos os trinta exemplares do corpus essa seção foi produzida, demonstrando a pertinência dessa unidade para a construção do tipo de artigo ora estudado. A unidade de Metodologia, no corpus investigado, foi elaborada a partir de cinco movimentos, aproximando-se de quatro movimentos construídos por Oliveira (2003), de um passo designado por Nwogu (1997) e de um movimento proposto por Costa (2015). Com o objetivo de apresentar a descrição dos artigos por meio dos movimentos e passos propostos pelas descrições desses autores, observemos, no Quadro 2, como ocorre a configuração das unidades informacionais na unidade de Metodologia do corpus:

QUADRO 2 - Frequência de unidades informacionais na seção de Metodologia em artigos empíricos da cultura disciplinar da área de Psicologia ${ }^{17}$

\begin{tabular}{|l|c|}
\hline \multicolumn{1}{|c|}{$\begin{array}{l}\text { UNIDADES INFORMACIONAIS DESCRITAS POR } \\
\text { OLIVEIRA (2003) }\end{array}$} & $\begin{array}{c}\text { Percentual atingido no } \\
\text { corpus investigado na área } \\
\text { de Psicologia }\end{array}$ \\
\hline $\begin{array}{l}\text { Movimento 1: Descrição do corpus ou dos participantes da } \\
\text { pesquisa } \\
\text { Passo 1 - Especificação do tamanho da amostra (tamanho } \\
\text { do corpus ou número de participantes) }\end{array}$ & $\mathbf{7 3 , 3 3 \%}$ \\
\hline Passo 2 - Especificação do perfil dos participantes & $\mathbf{7 3 , 3 3 \%}$ \\
\hline Passo 2A - Especificação do sexo e idade & $\mathbf{6 0 \%}$ \\
\hline $\begin{array}{l}\text { Passo 2B - Especificação do nível de escolaridade (estudantes, } \\
\text { professores etc.) }\end{array}$ & $46,67 \%$ \\
\hline
\end{tabular}

\footnotetext{
${ }^{16}$ Vale salientar que este estudo se identifica com as pesquisas de Abreu (2016), Bernardino e Abreu (2017) e Bernardino e Pacheco (2017), visto que esses tiveram como objetivo a descrição de uma seção retórica do gênero artigo a partir de distintas culturas disciplinares. ${ }^{17}$ Destacamos em negrito os movimentos e passos que efetivamente contribuíram para a recorrência da descrição sociorretórica final da unidade de Metodologia em artigos empíricos da cultura disciplinar da área de Psicologia.
} 


\begin{tabular}{|c|c|}
\hline $\begin{array}{l}\text { Passo } 2 \mathrm{C} \text { - Especificação da subárea a que os participantes } \\
\text { pertencem }\end{array}$ & $3,33 \%$ \\
\hline $\begin{array}{l}\text { Passo 2D - Especificação do nível de conhecimento dos } \\
\text { participantes na língua ou no tópico que está sendo investigado } \\
\text { pela pesquisa }\end{array}$ & $0 \%$ \\
\hline Passo 3 - Especificação do corpus selecionado & $10 \%$ \\
\hline $\begin{array}{l}\text { Movimento 2: Descrição dos materiais ou instrumentos } \\
\text { utilizados na coleta de dados }\end{array}$ & $93,33 \%$ \\
\hline Movimento 3: Descrição dos procedimentos & $93,33 \%$ \\
\hline Movimento 4: Descrição da análise dos dados & $66,67 \%$ \\
\hline $\begin{array}{l}\text { UNIDADE INFORMACIONAL DESCRITA } \\
\text { POR NWOGU (1997) }\end{array}$ & $\begin{array}{l}\text { Percentual atingido no } \\
\text { corpus investigado na área } \\
\text { de Psicologia }\end{array}$ \\
\hline $\begin{array}{l}\text { Movimento } 4 \text { - Descrevendo procedimentos de coleta de } \\
\text { dados } \\
\text { (1) Indicando fonte de dados }\end{array}$ & $60 \%$ \\
\hline $\begin{array}{l}\text { UNIDADE INFORMACIONAL DESCRITA } \\
\text { POR COSTA (2015) }\end{array}$ & $\begin{array}{l}\text { Percentual atingido no } \\
\text { corpus investigado na área } \\
\text { de Psicologia }\end{array}$ \\
\hline Movimento 4: Indicando aprovação por comitê de ética & $53,33 \%$ \\
\hline
\end{tabular}

Fonte: Nwogu (1997, p. 135), Oliveira (2003, p. 153) e Costa (2015, p. 195).

A partir da análise do Quadro 2, percebemos que o Movimento 1 elaborado por Oliveira (2003), Descrição do corpus ou dos participantes da pesquisa, apresentou recorrência a partir de três passos: Especificação do tamanho da amostra (tamanho do corpus ou número de participantes), Especificação do perfil dos participantes e Especificação do sexo e idade. As recorrências desses passos no corpus analisado foram, respectivamente, de 73,33\%, 73,33\% e 60\%. Também no corpus deste estudo, encontramos o passo Indicando a fonte de dados, com 60\% de recorrência, o qual é proveniente do movimento Descrevendo procedimentos de coleta de dados, construído por Nwogu (1997). Em relação ao Movimento 4, de Costa (2015), Indicando aprovação por comitê de ética, encontramos a recorrência de 53,33\% nos artigos analisados. Vejamos, no Quadro 3, como ficou configurada a seção de Metodologia no corpus investigado. 
QUADRO 3 - Descrição retórica da unidade de Metodologia em artigos empíricos da cultura disciplinar da área de Psicologia

\author{
Movimento 1: Descrevendo a amostra da pesquisa \\ Passo 1 - Especificando o tamanho da amostra \\ Passo 2 - Caracterizando o perfil dos participantes da amostra \\ Passo 3 - Indicando a fonte de dados \\ Movimento 2: Descrevendo os materiais ou instrumentos utilizados \\ na coleta de dados \\ Movimento 3: Descrevendo procedimentos experimentais \\ Movimento 4: Indicando aprovação por comitê de ética \\ Movimento 5: Descrevendo a análise de dados
}

Fonte: elaborado pelos autores conforme os movimentos e passos propostos por Nwogu (1997), Oliveira (2003) e Costa (2015).

De acordo com o Quadro 3, percebemos que o Movimento 1, Descrevendo a amostra da pesquisa, é segmentado em três passos: Passo 1 Especificando o tamanho da amostra; Passo 2 - Caracterizando operfil dos participantes da amostra; e Passo 3 - Indicando a fonte de dados. Adaptamos o modelo de Oliveira (2003) quanto aos passos 2, 2A, 2B, 2C e 2D (Quadro 1), visto que a descrição da autora aborda as características do perfil dos participantes das pesquisas nesses cinco passos - dentre eles, apenas o 2 e o $2 \mathrm{~A}$ foram recorrentes. Assim, acreditamos que juntá-los em apenas um passo reuniria essas informações e poderia designar claramente tais características, facilitando sua identificação.

De modo geral, o Movimento 1 descreve a quantidade da amostra presente no estudo bem como as características específicas desses participantes e a fonte de coleta dos dados, que, por sua vez, também é relacionada à amostra. O Passo 1 do movimento em questão, denominado Especificando o tamanho da amostra, que foi adaptado da proposta de Oliveira (2003), é caracterizado por apresentar apenas a dimensão da amostra envolvida na pesquisa (exemplos de 1 a 4), não sendo recorrente a especificação da dimensão do corpus, posto que só $10 \%$ dos artigos analisados investigaram corpus, em oposição a 90\% que contaram com participantes no estudo. 
(1) Para este estudo específico, foram selecionados $\mathbf{5 2 9}$ estudantes que responderam afirmativamente a, pelo menos, um dos itens do instrumento que indicavam violência na família e/ou na comunidade. (AAEP05)

(2) Participaram deste estudo sete profissionais, de diferentes áreas, atuantes nos três níveis de atenção do Sistema Único de Saúde (SUS). (AAEP07)

(3) Foram colaboradoras da pesquisa 10 grávidas, com idades entre 23 e 33 anos, captadas em um serviço de obstetrícia de um hospital privado de Brasília. (AAEP08)

(4) Participaram do estudo 122 mães de crianças portadoras de PC, que recebiam tratamento na Associação Mineira de Reabilitação (AMR) em Belo Horizonte-MG. (AAEP11)

No que concerne ao segundo passo, Caracterizando operfil dos participantes da amostra, são apontados detalhes da amostra do estudo. Encontram-se, neste passo do nosso corpus, apenas informações voltadas ao perfil dos participantes envolvidos na pesquisa. Essas informações destacam as particularidades da amostra quanto ao sexo e à idade, nível de escolaridade e até à subárea a que os participantes pertencem, corroborando o que orienta o manual da APA (2010), no que diz respeito à necessidade de identificar os participantes da pesquisa, detalhando características como sexo, nível de escolaridade etc. Esse passo também está em sintonia com o periódico Estudos de Psicologia (2016), que justifica que informações consistentes sobre os participantes devem ser evidenciadas. Esse achado pode ser percebido nos exemplos de 5 a 8 .

(5) A amostra deste estudo foi constituída por 429 alunos regularmente matriculados em diversos cursos, das áreas de Exatas, Humanas e Biológicas, de uma universidade particular do interior paulista. Do total de alunos, $172(40,1 \%)$ eram do sexo masculino e 257 $(59,9 \%)$ do sexo feminino. A idade variou entre 18 anos a 44 anos $(\mathbf{M}=22,7 ; \mathrm{DP}=4,85)$. Na distribuição por áreas do conhecimento, houve predominância para a área de Ciências Exatas, com frequência de 235 (54,8\%), seguida das Ciências Humanas, com 116 (27\%) e Ciências Biológicas com 78 (18,2\%). (AAEP02) 
(6) A amostra foi composta por 17 familiares (cuidadores informais) de pacientes oncológicos, incluindo adulto e infantil, e 16 cuidadores formais, ou seja, a equipe de profissionais do Hemocentro (enfermeiro, auxiliares de enfermagem, psicóloga, assistente social, médicos, pedagoga e farmacêutico). (AAEP13)

(7) Participaram deste estudo 47 graduandos cursando o $5^{\circ}$ e o $7^{\circ}$ semestres do curso de Psicologia de uma faculdade particular no interior do Estado de São Paulo, oito homens e 39 mulheres, com idade média de $\mathbf{2 6}$ anos e mediana de 23. (AAEP14)

(8) Participaram do estudo 120 crianças de ambos os sexos, faixa etária entre 6 e 12 anos $(M=9,46 ; D P=1,42)$, encaminhadas a um serviço de neurologia com queixas de dificuldades de aprendizagem para avaliação interdisciplinar. (AAEP18)

O terceiro passo, designado Indicando a fonte de dados, caracteriza o local a partir de onde foram coletados os dados das amostras dos estudos. É necessário enfatizar que esse passo apenas fornece informações referentes ao local onde os dados foram apurados, não sendo recorrentes informes acerca das datas em que ocorreram o levantamento desses dados (exemplos de 9 a 12). Nesse passo, recebem destaque informações referentes a localidades a partir da explicitação de cidades ou de locais, como escolas, hospitais, universidades etc.

(9) Participaram do estudo 122 mães de crianças portadoras de PC, que recebiam tratamento na Associação Mineira de Reabilitação (AMR) em Belo Horizonte-MG. (AAEP11)

(10) A pesquisa foi realizada em um Hemocentro de município de médio porte do interior de São Paulo. (AAEP13)

(11) Foi recrutada uma amostra não probabilística de conveniência com 491 indivíduos do sexo feminino $(n=282 ; 57,4 \%)$ e masculino $(n=209$; 42,6\%) na cidade de Porto Alegre/RS. (AAEP27)

(12) Participaram da pesquisa 615 estudantes de ensino médio, 149 professores e 32 funcionários de seis escolas públicas gaúchas, nas cidades de Porto Alegre, Venâncio Aires e Santa Cruz do Sul. (AAEP28)

O movimento 2, Descrevendo os materiais ou instrumentos utilizados na coleta de dados, recorrente em 93,33\% do corpus, é evidenciado por apontar e 
descrever quantos e quais foram os instrumentos e/ou materiais utilizados para colher os dados para o progresso do estudo (exemplos de 13 a 16).

(13) Entre as abordagens possíveis na pesquisa qualitativa optou-se pelo uso de entrevistas semiestruturadas para a produção de dados, uma vez que se trata de uma técnica flexível, que permite tanto adicionar questões quanto explorar novos pontos necessários conforme os objetivos propostos (Schraiber, 1995). (AAEP01)

(14) Para avaliação dos aspectos psicológicos, emocionais e saúde geral das mães utilizaram-se os instrumentos: Inventário de Depressão de Beck, Questionário de Estresse para Pais de Crianças com Transtornos do Desenvolvimento (QE-PTD) e Questionário de Saúde Geral. (AAEP11)

(15) Um Questionário Sociodemográfico foi usado para recolher informações acerca da idade, estado civil, escolaridade e situação profissional das participantes, além disso, foi utilizada a versão portuguesa do Adverse Childhood Experiences (ACE) Study Questionnaire (Felitti et al., 1998; Silva \& Maia, 2008), a qual inclui questões de autorrelato acerca de 10 categorias de experiências adversas divididas em duas áreas: experiências sobre as quais a criança é vítima direta (abuso emocional, físico e sexual e negligência física e emocional) e experiências integradas num contexto familiar disfuncional (familiares consumidores de substâncias, transtorno mental ou suicídio na família, prisão de um membro familiar, violência doméstica contra a figura materna e divórcio entre os pais). (AAEP19)

(16) O instrumento utilizado foi o Inventário de Comportamentos para Crianças e Adolescentes - CBCL 6 a 18 anos (Achenbach \& Rescorla, 2001), versão brasileira (Bordin, Mari, \& Caeiro, 1995), que avalia problemas de comportamento e competência social, sendo esta última obtida por três escalas (atividades, sociabilidade e escolaridade). (AAEP23)

Com base na APA (2010), na seção da Metodologia, é indispensável a descrição de todos os materiais ou instrumentos utilizados para a coleta de dados. É preciso apontar qual tipo de material foi empregado - como entrevistas, escalas, inventários e questionários -, detalhando cada um desses materiais ou instrumentos. No caso do corpus deste estudo, há alguns exemplares de artigos empíricos que pormenorizam com detalhes como e por quem o material foi elaborado, se foi adaptado, se é uma versão nacional 
ou internacional, qual o propósito de utilizá-lo, entre outras informações. Em alguns casos, esses instrumentos são apresentados em subtópicos ou, quando não o são, descritos no corpo do texto. Corroboramos com o argumento de Motta-Roth e Hendges (2010), de que informar sobre os materiais e instrumentos utilizados é uma importante função retórica da unidade de Metodologia.

Em relação às orientações das revistas do corpus para a construção da seção de Metodologia, as que tecem recomendações solicitam que sejam designados os instrumentos e materiais empregados no desenvolvimento da pesquisa. Quase todos os colaboradores entrevistados concordaram que esse é um aspecto fundamental a ser mencionado na unidade retórica em questão.

O terceiro movimento, Descrevendo procedimentos experimentais, demonstra o processo experimental, buscando explicar como ocorreu o desenvolvimento da pesquisa, apresentando o passo a passo de como foram aplicados os instrumentos, como foi feita a coleta de dados e o detalhamento desse processo. Esse passo alcançou 93,33\% de recorrência, sendo encontrado em 28 dos 30 artigos analisados. Para Oliveira (2003), no referido passo, há uma descrição do contexto em que a pesquisa está inserida. Consoante a autora, essa característica é mais visível em pesquisas qualitativas, entretanto, observamos que nos artigos resultantes de pesquisas quantitativas tal fato também foi bastante perceptível. Vejamos, a seguir, os exemplos que identificam esse passo (17 a 20).

(17) Num primeiro momento, foi aplicado o TDE seguindo-se as normas do manual. Na última etapa, foram aplicadas as tarefas de consciência fonológica - o teste de Spoonerismo, que consiste em fazer uma espécie de jogo com os sons das palavras. (AAEP16)

(18) As crianças foram incluídas no estudo mediante a permissão dos pais, registrada em Termo de Consentimento Livre e Esclarecido (TCLE). Para composição da amostra, foram levantados resultados de avaliações do banco de dados do Laboratório de Distúrbios, Dificuldades de Aprendizagem e Transtorno da Atenção (Disapre), organizados conforme os objetivos da pesquisa. (AAEP18)

(19) Os dados foram coletados nas salas de aula durante o período letivo regular universitário, em cursos de graduação e pós-graduação, visando pessoas com maior idade e relações amorosas mais estáveis. Também foram abordados participantes em projetos universitários de extensão comunitária, em associações e grupos de casais diversos, buscando dados em setores variados da sociedade. (AAEP27) 
(20) Na condição controle, o questionário era respondido apenas uma vez de forma individual, imediatamente após a visualização do vídeo. Ao final do procedimento, foi esclarecido aos participantes o objetivo da pesquisa, sendo explicado, na condição concordância, a atuação do confederado, garantindo que o participante não havia desconfiado da encenação durante o procedimento. (AAEP29)

Em concordância com o que menciona o colaborador 8 , o passo a passo do estudo deve ser detalhado, sendo imprescindível que, nessa seção, sejam explanados os procedimentos das etapas da pesquisa. No manual da APA (2010) também consta a importância do fornecimento de informações acerca dos procedimentos de seleção da amostra, como eles foram agrupados para a coleta de dados e como esses momentos ocorreram. Todas essas etapas devem ser detalhadas, pontua o manual.

O Movimento 4, Indicando aprovação por comitê de ética, indica, nos artigos empíricos, que a pesquisa foi aprovada por um comitê de ética. É preciso salientar que esse movimento recebe destaque em diferentes casos, seja na subseção dos procedimentos, numa subseção própria ou no texto corrido, que é quando não há subseções destacadas no corpo do artigo (ver exemplos de 21 a 24).

(21) O estudo foi submetido e aprovado por dois Comitês de Ética em Pesquisa da: Secretaria Municipal de Saúde (CEP-SMS) de São Paulo (Parecer no 52/10) e da Faculdade de Medicina e complexo Hospital das Clínicas (CAPPesq; Protocolo n 1075/08). (AAEP01)

(22) O presente trabalho foi aprovado pelo Comitê de Ética em Pesquisa (CEP) da Universidade Católica de Brasília-UCB, em $\mathrm{Abr} / 2011$, sob o $\mathrm{n}^{\circ}$ do parecer 085/2011. (AAEP08)

(23) O projeto deste estudo foi aprovado pelo Comitê de Ética em Pesquisa da Pontifícia Universidade Católica de Campinas, instituição a que se vinculam as autoras, por meio do parecer número 375/09, atendendo aos padrões estabelecidos pela Resolução 196/96. (AAEP14)

(24) Este trabalho foi aprovado pelo Comitê de Ética em Pesquisa da Faculdade de Ciências Médicas da Universidade Estadual de Campinas - Unicamp (Protocolo $n^{\circ}$ 476.243). (AAEP18) 
O Movimento 4, Indicando a aprovação por comitê de ética, percebido em $53,33 \%$ do corpus, estabelece qual é o comitê de ética específico que aprovou a pesquisa, também apresentando números de processos, protocolos, pareceres, resoluções etc. O periódico Temas em Psicologia (2016) evidencia que todos os artigos que tenham realizado procedimentos envolvendo seres humanos na coleta de dados devem conter informações acerca do parecer favorável de algum Comitê de Ética em Pesquisa (CEP) vinculado à Comissão Nacional de Ética em Pesquisa (Conep). A revista Estudos de Psicologia (2016) também requisita que recebam destaque informações relacionadas à aprovação dos comitês de ética. Contudo, percebemos que alguns exemplares de artigos em que a pesquisa envolve seres humanos não apresentaram informações referentes à aprovação por comitês. Talvez a omissão dessas informações nos artigos se deva à falta de orientação de quase todos os periódicos e do guia da APA (2010). Mesmo sem essas orientações, 53,33\% do corpus apresentaram recorrência para essa unidade informacional, ou seja, os autores desses estudos consideraram relevante realçar essas informações.

O quinto e último movimento, Descrevendo a análise de dados, trata de discutir como são feitas as análises e interpretações dos dados coletados. No corpus deste trabalho, observamos que, nesse movimento, são apontados como os dados serão analisados e, em alguns casos, destacam-se os tipos de análise empregados e os programas utilizados (exemplos de 25 a 28).

(25) Do ponto de vista da interpretação, os dados da pesquisa foram analisados através da análise de conteúdo do tipo temático. Esse tipo de análise visa compreender o pensamento do sujeito por meio do conteúdo de suas falas e de depoimentos em texto e permite tanto aprofundar os temas que foram propostos pelo roteiro de entrevista quanto identificar temas emergentes (Schraiber, 1995; Caregnato; Mutti, 2006; Minayo, 2008). (AAEP01)

(26) A análise dos dados foi realizada por meio do software SPSS, versão 18. Por meio de análises descritivas, foram avaliadas frequências e porcentagens das respostas dos itens do instrumento relativos à vivência de situações de violência no ambiente familiar e comunitário, bem como comparadas as médias, por meio de testes $t$ de Student, dos diferentes tipos de abusos vivenciados por adolescentes e jovens. (AAEP05) 
(27) Os dados foram analisados em um pacote estatístico Statistical Package of Social Science versão 11.5, seguindo os seguintes passos: 1) análise descritiva dos resultados sobre estado emocional das mães e sobre o comportamento das crianças com PC;2) análise de correlação, utilizando o coeficiente de Pearson; 3) regressão múltipla, utilizando o método stepwise. (AAEP11)

(28) As análises estatísticas foram realizadas utilizando-se o programa Statistical Package for the Social Sciences (SPSS), versão 17. (AAEP12)

Segundo Motta-Roth e Hendges (2010), a função retórica da Metodologia é narrar os procedimentos de coleta e análise dos dados e descrever os materiais que possibilitaram a obtenção de resultados. O colaborador 8 concorda com o que disseram as autoras ao enfatizar que nesse movimento é crucial indicar como os dados foram analisados. Os periódicos Estudos e Pesquisas em Psicologia (2016), Psicologia e Sociedade (2016) e Temas em Psicologia (2016) julgam indispensável realizar a análise dos dados na seção de Metodologia.

De maneira geral, a unidade retórica de Metodologia é uma seção curta e suficientemente informativa. Ela apresenta, em 60\% dos artigos analisados, subseções destacadas para identificar as unidades retóricas, tais como: participantes, materiais e instrumentos, procedimentos, análise de dados etc. Os 40\% restantes dos artigos apresentam as unidades retóricas diluídas no corpo do texto, solicitando ao leitor maior atenção para as pistas léxico-gramaticais indicadoras das funções retóricas.

Outro ponto relevante é que há distintas terminologias para denominar a seção de Metodologia. A nomenclatura mais comum é Método, encontrada em 76,67\% do corpus. Entre as outras nomenclaturas, podemos mencionar: Metodologia (AAEP01, AAEP25); Percurso metodológico (AAEP04); A pesquisa (AAEP06); Procedimentos metodológicos (AAEP07); Procedimento de configuração do acontecer (AAEP14) e Itinerários da incursão ao outro (AAEP26). Acreditamos que, como o guia da APA (2010) e algumas revistas investigadas pouco versam sobre recomendações quanto a essa nomenclatura, os autores sentem-se livres para construir diferentes terminologias. 


\subsection{Apresentando as pistas léxico-gramaticais da unidade retórica de Metodologia}

Para encerrarmos a análise da unidade retórica aqui estudada, apresentamos as pistas léxico-gramaticais das unidades informacionais presentes nos artigos investigados. Vejamos no Quadro 4:

QUADRO 4 - Apresentando as pistas léxico-gramaticais da unidade retórica de Metodologia

\begin{tabular}{|c|c|}
\hline \multicolumn{2}{|c|}{$\begin{array}{l}\text { Movimento 1: Descrevendo a amostra da pesquisa } \\
\text { Passo 1 - Especificando o tamanho da amostra }\end{array}$} \\
\hline Tipo de item & Exemplos \\
\hline $\begin{array}{l}\text { Numerais indicando dimensão da } \\
\text { amostra }\end{array}$ & $429,1.140,32,54,3,22,100,7$ \\
\hline $\begin{array}{l}\text { Expressão indicativa da função } \\
\text { retórica }\end{array}$ & $\begin{array}{l}\text { Amostra, amostra final, amostra não probabilística de } \\
\text { conveniência, amostra foi não probabilística. }\end{array}$ \\
\hline \multicolumn{2}{|c|}{ Passo 2-Caracterizando o perfil dos participantes da amostra } \\
\hline Tipo de item & Exemplos \\
\hline $\begin{array}{l}\text { Expressão definidora dos } \\
\text { participantes envolvidos }\end{array}$ & $\begin{array}{l}\text { Adolescentes, alunos, casais, crianças, crianças de } \\
\text { baixa renda, cuidadores formais, cuidadores informais, } \\
\text { estudantes, estudantes de ensino médio, estudantes } \\
\text { universitários, familiares, funcionários de escolas, } \\
\text { graduandas/os, grávidas, habitantes de um conjunto } \\
\text { residencial, indivíduos, mães, mulheres, professores, } \\
\text { profissionais de diferentes áreas, psicólogos, sujeitos, } \\
\text { travestis. }\end{array}$ \\
\hline \multicolumn{2}{|c|}{ Passo 3 - Indicando a fonte de dados } \\
\hline Tipo de item & Exemplos \\
\hline Expressão adverbial de lugar & $\begin{array}{l}\text { Em escolas públicas, em um Hemocentro de município } \\
\text { de médio porte do interior de São Paulo; na Associação } \\
\text { Mineira de Reabilitação (AMR) em Belo Horizonte, } \\
\text { na cidade de Porto Alegre, na cidade de São Paulo, na } \\
\text { cidade do Recife, nas cidades de Porto Alegre, Venâncio } \\
\text { Aires e Santa Cruz do Sul, entre outros. }\end{array}$ \\
\hline $\begin{array}{l}\text { Locução verbal indicativa da } \\
\text { função retórica, local onde os } \\
\text { dados coletados foram realizados } \\
\text { e/ou estavam inseridos }\end{array}$ & $\begin{array}{l}\text { [A amostra] foi constituída por, [A pesquisa] foi } \\
\text { realizada em, Foi recrutada [uma amostra] na... }\end{array}$ \\
\hline
\end{tabular}




\begin{tabular}{|c|c|}
\hline Tipo de item & Exemplos \\
\hline $\begin{array}{l}\text { Grupos nominais indicativos de } \\
\text { instrumento }\end{array}$ & $\begin{array}{l}\text { Entrevista, escala, ficha de catalogação, ficha de } \\
\text { contato, ficha de dados demográficos, instrumento, } \\
\text { inventário, lista, medida, pós-teste, pré-teste, protocolo, } \\
\text { questionário sociodemográfico, questionário, roteiro de } \\
\text { entrevista, subteste, teste. }\end{array}$ \\
\hline Voz passiva: & $\begin{array}{l}\text { Foi: aplicado(a), elaborado, examinada, usado, utilizado. } \\
\text { Foram: obtidos, realizadas, utilizados, realizadas. } \\
\text { Aplicou-se, optou-se, utilizaram-se, utilizou-se. }\end{array}$ \\
\hline \multicolumn{2}{|c|}{ Movimento 3: Descrevendo procedimentos experimentais } \\
\hline Tipo de item & Exemplos \\
\hline $\begin{array}{l}\text { Voz passiva/verbos que indicam a } \\
\text { realização de procedimentos }\end{array}$ & $\begin{array}{l}\text { Foi: aplicado, apresentado, assinado, conduzida, } \\
\text { divulgado, efetuado, esclarecido, implantado, } \\
\text { inspecionada, investigada, investigado, observado, } \\
\text { realizado(a), solicitado, utilizado. } \\
\text { Foram: abordados, agendados, analisados, aplicadas, } \\
\text { aplicados, apresentados(as), assinados, atendidos, } \\
\text { audiogravadas, avaliados, classificados(as), coletados, } \\
\text { convidados(as), destacados, divididas, efetuadas, } \\
\text { elaboradas, feitas, garantidos, gravadas, incluídas, } \\
\text { instruídos, introduzidos, levantados, lidos, preenchidos, } \\
\text { realizados(as), recolhidas, resumidos, selecionados, } \\
\text { submetidos, transcritas, utilizados(as). } \\
\text { Era: apresentado, solicitado. } \\
\text { Eram: apresentados, fornecidos. } \\
\text { Aplicou-se, criou-se, explicitou-se, focalizou-se, } \\
\text { realizou-se, recorreu-se. }\end{array}$ \\
\hline \multicolumn{2}{|c|}{ Movimento 4: Indicando aprovação por comitê de ética } \\
\hline Tipo de item & Exemplos \\
\hline $\begin{array}{l}\text { Expressão denotativa de função } \\
\text { retórica }\end{array}$ & $\begin{array}{l}\text { Foi: aprovado(a), submetido e aprovado, submetida e } \\
\text { aprovada. } \\
\text { Aprovação do Comitê de Ética em Pesquisa. }\end{array}$ \\
\hline $\begin{array}{l}\text { Expressão denotativa do Registro } \\
\text { do estudo em comitê de ética }\end{array}$ & Parecer, processo, protocolo, resolução. \\
\hline $\begin{array}{l}\text { Expressão denotativa de } \\
\text { consentimento dos participantes } \\
\text { envolvidos na pesquisa }\end{array}$ & Termo de Consentimento Livre e Esclarecido. \\
\hline
\end{tabular}




\begin{tabular}{|l|l|}
\hline \multicolumn{2}{|c|}{ Movimento 5: Descrevendo a análise de dados } \\
\hline \multicolumn{1}{|c|}{ Tipo de item } & \multicolumn{1}{c|}{ Exemplos } \\
\hline $\begin{array}{l}\text { Expressão denotativa de função } \\
\text { retórica }\end{array}$ & $\begin{array}{l}\text { Análise de correlação, análise descritiva e inferencial, } \\
\text { análise descritiva, análise dos dados, análise exploratória, } \\
\text { análise interpretativa, análise mista dos resultados, } \\
\text { análise qualitativa dos dados, análises estatísticas. }\end{array}$ \\
\hline Voz passiva & $\begin{array}{l}\text { Foi: administrado, efetuado, feita, produzida, realizada, } \\
\text { realizado, usado, utilizado, } \\
\text { Foram: analisados, avaliados(as), comparadas, } \\
\text { considerados, identificadas, introduzidos, organizados, } \\
\text { realizadas, submetidos(as), usados. } \\
\text { Adotou-se, efetuou-se, fez-se, optou-se, procedeu-se, } \\
\text { realizou-se, recorreu-se. } \\
\text { Apresentaram, revelou. }\end{array}$ \\
\hline
\end{tabular}

Fonte: elaborado pelo autor com base em Pacheco (2016), com a inclusão de características específicas da cultura disciplinar da área de Psicologia.

O Movimento 1, Descrevendo a amostra da pesquisa, apresenta como função retórica caracterizar a amostra a partir de expressões lexicais como "amostra", "amostra final", entre outras designações. Utilizaram-se conceitos que definiam os participantes envolvidos nos estudos e expressões adverbiais que indicavam os locais em que as pesquisas foram realizadas.

O Movimento 2, Descrevendo os materiais ou instrumentos utilizados na coleta de dados, apresenta os materiais ou instrumentos utilizados na coleta de dados. No que se refere à voz passiva observada no emprego desse movimento, para Swales (1990), sua utilização é comum na descrição da unidade de Metodologia. O uso desse recurso pode estar relacionado ao ocultamento da agência do pesquisador, transferindo essa agência para os próprios procedimentos da pesquisa.

No Movimento 3, Descrevendo procedimentos experimentais, destacam-se os verbos ou locuções verbais que se encontram na voz passiva e que indicam a realização de procedimentos da pesquisa. As expressões mais observadas foram: "foi aplicado", "foram apresentados(as)" e "aplicou-se".

O Movimento 4, Indicando aprovação por comitê de ética, designa que as pesquisas foram aprovadas por respectivos comitês e menciona expressões que caracterizam essa aprovação, como "processo", "parecer", "protocolo" e "resolução". A função retórica desse movimento foi claramente percebida a partir de expressões utilizadas pelos autores dos artigos, sendo recorrentes, nesse caso, expressões lexicais como "foi submetido(a) e aprovado(a)". 
Em relação ao Movimento 5, Descrevendo a análise de dados, são apresentadas informações referentes aos tipos de análise utilizados na pesquisa, como verbos na voz passiva que expressam processos que envolvem tais análises. Como exemplos dessas expressões, podemos apontar: "foram analisados" e "foram avaliados(as)".

Dessa forma, no que tange à configuração sociorretórica da unidade de Metodologia, percebemos que essa seção é iniciada pela descrição da amostra quanto a sua dimensão, características da amostra e designação dos locais em que foram realizadas as coletas de dados. $\mathrm{Na}$ sequência, são discutidos os materiais ou instrumentos empregados no estudo, depois, são apontados os procedimentos experimentais. Informações que relatam a aprovação das pesquisas por comitês de ética também foram recorrentes no corpus. Por fim, é descrito o procedimento da análise de dados.

\section{Considerações finais}

Este estudo e os demais realizados no âmbito do grupo de pesquisa Dileta têm como objetivo investigar como as características das distintas culturas disciplinares influenciam a produção dos gêneros acadêmicos. Acreditamos que, para ser possível a compreensão de como determinado gênero acadêmico é concebido, distribuído e compreendido, é preciso investigar as culturas disciplinares, pois apenas assim haverá possibilidade de compreensão global sobre como as crenças, valores, questões epistemológicas, entre outros aspectos que identificam a cultura, influenciam a maneira como seus membros constroem os gêneros acadêmicos.

Nesta pesquisa, em específico, nos dedicamos ao estudo da cultura disciplinar da área de Psicologia, evidenciando a produção da unidade retórica de Metodologia em artigos acadêmicos empíricos desta área. Como achados, compreendemos que a área de Psicologia preza por elaborar, em artigos empíricos, informações que caracterizem a amostra da pesquisa, apontem os materiais e instrumentos utilizados, descrevam os procedimentos, mencionem a aprovação de pesquisas com seres humanos mediante número de processos admitidos por comitês de ética e, por fim, informações que descrevam como ocorre a análise de dados. Vale ressaltar, caracterizando a perspectiva sociorretórica desse estudo, que há correspondência entre os achados desta pesquisa com os dados encontrados no manual da APA (2010), as orientações dos periódicos e das informações disponibilizadas pelos membros experientes da área, nos levando a entender 
a pertinência da descrição sociorretórica na investigação do gênero artigo acadêmico.

Destarte, a maior implicação deste estudo diz respeito ao ensino da escrita acadêmica na universidade, o qual, na maioria das vezes, toma como parâmetro apenas as orientações de associações reguladoras de normas para formatação de trabalhos acadêmicos, negligenciando as características próprias das culturas disciplinares, ignorando, portanto, a relação entre as características dos gêneros e do grupo social que os utiliza. Pretendemos, assim, a partir desta pesquisa, contribuir para o letramento acadêmico na área de Psicologia em relação ao modo como o artigo acadêmico empírico é produzido e compreendido.

\section{Agradecimentos}

Agradecemos à Coordenação de aperfeiçoamento de Pessoal de Nível Superior (Capes) pela concessão de bolsa de mestrado acadêmico para a realização deste estudo.

\section{Referências}

ABREU, N. O. O artigo acadêmico na cultura disciplinar da área de Psicologia: um estudo sociorretórico. 2016. 213 f. Dissertação (Mestrado em Linguística Aplicada) Programa de Pós-Graduação em Linguística Aplicada, Universidade Estadual do Ceará, Fortaleza, 2016.

AMERICAN PSYCHOLOGICAL ASSOCIATION. Publication manual of the American Psychological Association. Washington, DC: APA Style, 2010.

ASKEHAVE, I.; SWALES, J. M. Identificação de gênero e propósito comunicativo: um problema e uma possível solução. In: BEZERRA, B. G.; BIASI-RODRIGUES, B.; CAVALCANTE, M. M. (Org.). Gêneros e sequências textuais. Recife: Edupe, 2009, p. 221-247.

BERNARDINO, C. G. Artigo acadêmico: espaço de posicionamento e negociações. Polissema: Revista de Letras do Iscap, Porto, v. 6, p. 61-78, 2006.

BERNARDINO, C. G.; ABREU, N. O. A seção de introdução em artigos acadêmicos experimentais da cultura disciplinar de psicologia: um estudo sociorretórico. Raído, Dourados, v. 11, n. 27, p. 463-482, 2017.

BERNARDINO, C. G.; COSTA, R. L. S. A introdução de artigos acadêmicos e as diferenças entre culturas disciplinares. Intersecções, Jundiaí, ano 9, n. 1, p. 151-170, 2016. 
BERNARDINO, C. G.; PACHECO, J. T. S. Uma análise sociorretórica de introduções em artigos originais da cultura disciplinar da área de Nutrição. Fórum Linguístico, Florianópolis, v. 14, n. 1, p. 1749-1766, 2017. Doi: https:// doi. org/10.5007/1984-8412.2017v14n1p1749

BHATIA, V. K. A análise de gêneros hoje. Tradução Benedito Gomes Bezerra. In: BEZERRA, B. G.; BIASI-RODRIGUES, B.; CAVALCANTE, M. M. (Org.). Gêneros e sequências textuais. Recife: Edupe, 2009. p. 159-195.

CONSELHO REGIONAL DE PSICOLOGIA DA $6{ }^{a}$ REGIÃO. Exposição 50 anos dapsicologia no Brasil: a História da psicologia no Brasil. São Paulo, 2011. Disponível em: < https://bit.ly/2uhuNOb>. Acesso em: 23 mar. 2016.

COORDENAÇÃO DE APERFEIÇOAMENTO DE PESSOAL DE NÍVEL SUPERIOR. Documento de área 2013. Área de avaliação: Psicologia. Brasília, DF, 2013. Disponível em: <https://bit.ly/2KQ2sFz>. Acesso em: 4 mar. 2016.

COSTA, R. L. S. Culturas disciplinares e artigos acadêmicos experimentais: um estudo comparativo da descrição sociorretórica. 2015. 242 f. Dissertação (Mestrado em Linguística Aplicada) - Programa de Pós-Graduação em Linguística Aplicada, Universidade Estadual do Ceará, Fortaleza, 2015.

ESTUDOS DE PSICOLOGIA. Instruções aos autores. Campinas, 2016. Disponível em: < https://bit.ly/2ubSdUO>. Acesso em: 26 mar. 2016.

ESTUDOS E PESQUISAS EM PSICOLOGIA. Instrucões aos autores. Rio de Janeiro, 2016. Disponível em: <https://bit.ly/2KQw2uv>. Acesso em: 26 mar. 2016.

FÉRES-CARNEIRO, T. Memórias do curso de Pós-graduação em Psicologia da PUC-Rio: comemorando seus 40 anos. Psicologia Clínica, Rio de Janeiro, v. 19, n. 1, p. 217-225, 2007.

FRACTAL. Instruções aos autores. Rio de Janeiro, 2016. Disponível em: < https:/ / bit. ly/2NeZtr6>. Acesso em: 26 mar. 2016.

HYLAND, K. Disciplinary discourse: social interactions in academic writing. Singapura: Pearson Education Limited, 2000.

LIMA, M. V. B; ABREU, N. O. Letramento acadêmico: análise sobre como manuais didáticos de metodologia abordam o ensino do gênero artigo acadêmico. Entrepalavras, Fortaleza, v. 7, p. 9-25, jan./jun. 2017.

MOTTA-ROTH, D.; HENDGES, G. R. Produção textual na universidade. São Paulo: Parábola, 2010.

NWOGU, K. N. The Medical research paper: structure and functions. English for Specific Purposes, Amsterdam, v. 16, n. 2, p. 119-138, 1997. Doi: https://doi. org/10.1016/S0889-4906(97)85388-4 
OLIVEIRA, F. M. A configuração textual da seção de metodologia em artigos acadêmicos de Linguística Aplicada. 2003. 136 f. Dissertação (Mestrado em Letras) - Curso de PósGraduação em Letras, Universidade Federal de Santa Maria, Santa Maria.

PACHECO, J. T. S. O artigo acadêmico na cultura disciplinar da área de Nutrição: uma investigação sociorretórica. 2016. 201 f. Dissertação (Mestrado em Linguística Aplicada) - Programa de Pós-Graduação em Linguística Aplicada, Universidade Estadual do Ceará, Fortaleza, 2016.

PINHEIRO, M. V. B. L. Uma análise do gênero artigo acadêmico em manuais de orientação da escrita científica. 2016. 216 f. Dissertação (Mestrado em Linguística Aplicada) Programa de Pós-Graduação em Linguística Aplicada, Universidade Estadual do Ceará, Fortaleza, 2016.

PSICOLOGIA EM PESQUISA. Instruções aos autores. Juiz de Fora, 2016. Disponível em: < https://bit.ly/2zztGPK>. Acesso em: 26 mar. 2016.

PSICOLOGIA E SOCIEDADE. Instruçẽes aos autores. Belo Horizonte, 2016. Disponível em: < https://bit.ly/2NwJwh1>. Acesso em: 26 mar. 2016.

PSICOLOGIA: TEORIA E PESQUISA. Instruções aos autores. Brasília, DF, 2016. Disponível em: < https:/ / bit.ly/2KSYOL2>. Acesso em: 26 mar. 2016.

PSICO-USF. Instruções aos autores. Campinas, 2016. Disponível em: <https://bit. ly/2KR6Sfb >. Acesso em: 26 mar. 2016.

SAÚDE E SOCIEDADE. Instruções aos autores. São Paulo, 2016. Disponível em: <https://bit.ly/2us40j5>. Acesso em: 26 mar. 2016.

SAÚDE EM DEBATE. Instruções aos autores para preparação e submissão de artigos. Rio de Janeiro, [2016]. Disponível em: < https://bit.ly/2NPlOwA>. Acesso em: 26 mar. 2016.

SOARES, A. R. A psicologia no Brasil. Psicologia: Ciência e Profissão, Brasília, v. 30, p. 8-41, 2010. Número especial. Doi: https://doi.org/10.1590/S141498932010000500002

SWALES, J. M. Genre analysis: English in academic and research settings. Cambridge: Cambridge University Press, 1990.

SWALES, J. M. Research genres: explorations and applications. Cambridge: Cambridge University Press, 2004. Doi: https://doi.org/10.1017/CBO9781139524827

TEMAS EM PSICOLOGIA. Instruções aos autores. Ribeirão Preto, 2016. Disponível em: <https://bit.ly/2Kz2gOO>. Acesso em: 26 mar. 2016.

Data de submissão: 21/12/2017. Data de aprovação: 12/04/2018. 